\title{
The Clinical Efficacy of Reflexology in Nursing Home Residents with Dementia
}

\author{
NANCY A. HODGSON, Ph.D., R.N., ${ }^{1}$ and SUSAN ANDERSEN, B.S., C.H.E.S. ${ }^{2}$
}

\begin{abstract}
Objective: This experimental, repeated-measures, crossover design study with nursing home residents examined the efficacy of reflexology in individuals with mild-to-moderate stage dementia. Specifically, the study tested whether a weekly reflexology intervention contributed to the resident outcomes of reduced physiologic distress, reduced pain, and improved affect.

Setting: The study was conducted at a large nursing home in suburban Philadelphia.

Sample: The sample included 21 nursing home residents with mild-to-moderate stage dementia randomly assigned to two groups.

Interventions: The first group received 4 weeks of weekly reflexology treatments followed by 4 weeks of a control condition of friendly visits. The second group received 4 weeks of friendly visits followed by 4 weeks of weekly reflexology.

Outcome measures: The primary efficacy endpoint was reduction of physiologic distress as measured by salivary $\alpha$-amylase. The secondary outcomes were observed pain (Checklist of Nonverbal Pain Indicators) and observed affect (Apparent Affect Rating Scale).

Results: The findings demonstrate that when receiving the reflexology treatment condition, as compared to the control condition, the residents demonstrated significant reduction in observed pain and salivary $\alpha$-amylase. No adverse events were recorded during the study period.

Conclusions: This study provides preliminary support for the efficacy of reflexology as a treatment of stress in nursing home residents with mild-to-moderate stage dementia.

\section{INTRODUCTION}

$\mathbf{T}$ The anxiety associated with unfamiliar surroundings, loss of self-control, social isolation, and mental confusion that accompany Alzheimer's disease and related dementias often create stress for individuals living in the nursing home environment. This can affect the autonomic (sympathetic) nervous system and induce emotional and physical symptoms of distress. ${ }^{1}$ The growing body of anecdotal literature suggests that alternative and complementary therapies may be an effective treatment for older adults with dementia, appearing to relieve distress without the side-effects of pharmaceuticals. Complementary "touch" therapies such as massage and reflexology may reduce the experience of distress

for nursing home residents by promoting a relaxation response. ${ }^{2-4}$

Touch described as "gentle" and "supportive" can be beneficial to nursing home residents with Alzheimer's disease $^{5-7}$ Touch given with the intention of comfort is believed not only to communicate caring but also to have beneficial stress-reducing effects on body systems. ${ }^{8,9} \mathrm{Al}$ though research into the efficacy of touch therapies is taking priority, ${ }^{10,11}$ one of the common challenges in conducting literature reviews in the field of touch therapy is the lack of consistent terminology for describing the treatment. This absence of a common language makes it difficult to assess whether research protocols are reproducible.

A review of the literature on touch therapies specific to
\end{abstract}

\footnotetext{
${ }^{1}$ Center for Applied Research on Aging and Health, Thomas Jefferson University Philadelphia, PA.

${ }^{2}$ Polisher Research Institute (formerly the Philadelphia Geriatric Center), North Wales, PA.
} 
the older population reveals three critical omissions in the research to date. First, studies evaluating the effects of touch therapies have overlooked individuals in the later stages of dementia. Second, existing studies often lack an experimental design, so there is no comparison group, a crucial element in evaluating the effects of treatment. Third, and perhaps most important for the proposed study, evaluations of touch therapies have almost exclusively relied upon observational rather than physiologic measures.

Studies published in the 1970s and 1980s on "gentle, stroking touch" surmised that patients with dementia patients may be contraindicated for this technique due to their altered sensory perception. ${ }^{12,13}$ However, these studies were conducted with few subjects, and relied on observational measures of outcomes. Subsequent studies expanded their sample groups and continued to examine the efficacy of "slow stroke" massage in individuals with early-stage dementias. ${ }^{2,14,15}$ Four evaluations of gentle massage protocols in adults with moderate-stage dementias demonstrated decreased observed expressions of agitation such as pacing and resistance. ${ }^{6,16-18}$

Although reflexology and massage are often confused as similar modalities, there are several key differences between the modalities. Both involve the use of the hands to apply their techniques. However, reflexology uses small muscle movements applied to specific areas of the body (feet and hands), whereas massage uses large muscle movements and is often applied to the whole body. In reflexology, the pressure applied to the reflex points stimulates blood flow and nerve impulses, releases toxins and endorphins, and harmonizes physiologic function. ${ }^{19,20}$ Since reflexology training programs typically require less training (approximately 200 hours) than massage training (500 hours), and are less invasive and of shorter duration, they are often less costly than massage treatments.

Studies evaluating the effect of reflexology in a variety of health conditions have shown that it is a promising treatment in reducing pain and in promoting well-being with little risk of side-effects. ${ }^{10,11}$ In randomized controlled trials, reflexology has been found to be effective in reducing pain in women with severe premenstrual symptoms, ${ }^{21}$ and in patients with migraine and tension headaches. ${ }^{22}$ It has also demonstrated benefit in alleviating motor, sensory, and urinary symptoms in patients with multiple sclerosis. ${ }^{23}$ Recent systematic reviews on the efficacy of reflexology with patients with cancer found positive improvements in anxiety and pain. ${ }^{24-26}$ While these studies reflect promising and encouraging insight into the future of reflexology, there are limited data on how nursing home residents, specifically those with dementia, can benefit from receiving reflexology treatments.

\section{MATERIALS AND METHODS}

The project reported here was undertaken to test the efficacy of reflexology treatment as a complementary touch therapy in the treatment of distress in nursing home residents with dementia. It tests the following research question: "Does a weekly reflexology intervention contribute to nursing home resident outcomes of reduced distress, reduced pain, and improved affect, when compared to a control condition?"

\section{Study participants}

Nursing home residents were approached for inclusion into the study by Assistant Directors of Nursing at a 324bed nursing home in suburban Philadelphia. The medical director of the facility gave final approval to contact residents and their responsible party for consent. Subjects were included if they were residents of the nursing home for at least 6 months, over age 75, with a probable diagnosis of dementia, who were willing and able to participate for the duration of the study (signed consent or verbal assent). A probable diagnosis of dementia was determined by the Functional Assessment Staging scale criteria. ${ }^{27}$ Exclusion criteria were based on the relevant reflexology literature ${ }^{10,20}$ that outlines suitability for reflexology treatment. Residents were excluded if they suffered from the following conditions: history of deep vein thrombosis, epilepsy, bile or kidney stones, had a pacemaker, or had fever, open foot wounds, or foot fractures. Exclusion criteria also included recent hospitalization (less than 1 month) preceding or during the study period, and recent onset or discontinuation (less than 2 weeks) of physiotherapy that included massage therapy. Letters of consent and intervention schedules were also sent to the next of kin for additional approval of consent. The protocol and informed consent procedures were approved by the nursing home's Institutional Review Board.

A randomized, crossover design was selected because it offered advantages over parallel group trials including (1) that each participant acted as his or her own control, eliminating among-subject variation; (2) that fewer subjects were required to obtain the same power; and (3) that every subject received both the experimental and control condition. Consenting subjects were randomized into two conditions. Those assigned to the first group received 4 weeks of reflexology followed by 4 weeks of friendly visits. Those assigned to the second group received 4 weeks of friendly visits followed by 4 weeks of reflexology. To reduce the extraneous effects of multiple interventionists, the friendly visits and reflexology sessions were conducted by a single, certified, reflexology provider.

\section{Treatment condition}

The reflexology protocol was delivered by a trained reflexologist weekly for 4 weeks at the same time (midmorning) and on the same day each week. A 30-minute reflexology session is the recommended duration for vulnerable populations such as older adults and children. A typical treatment began with a progressive relaxation exercise, using the protocol described by Welden and Yesavage, ${ }^{28}$ and was fol- 
Table 1. Pressure Sequence of Treatment Condition Protocol

\begin{tabular}{lll}
\hline Area & \multicolumn{1}{c}{ Technique } & Reflex point \\
\hline Top of toes & Pivot & Head/brain \\
Balls of toes 2-5 & Pivot & Sinus \\
Base/sides of toes 2-5 & Thumb walking & Eyes/ears \\
Bottom/sides of great toe & Thumb walking & Head \\
Bottom/sides of great toe & Hook & Pituitary gland \\
Bottom/sides of great toe & Pivot & Thyroid \\
Tops of toes & Finger walking & Teeth \\
Lateral base of toe 5 & Pivot & Shoulder \\
Ridge under toes & Thumb walking & Neck, jaw, inner ear \\
Medial base of great toe & Pivot & Neck \\
Ball of foot & Thumb-walking (both directions) & Chest, lung, heart \\
Top of foot-between metatarsals & Single finger walking & Lymphatic \\
Top of foot & Finger walking & Lymphatic \\
Top of foot-between 1st \& 2nd & Pivot & Lymphatic drainage \\
metatarsal & Thumb walking-both directions & Upper abdomen \\
Upper arch & Thumb walking along colon & Colon \\
Lower arch & Thumb walking—both directions & Small intestines \\
Lower arch & Thumb walking or gentle knuckle & Pelvis \\
Heel & roll-both directions & Spine \\
Medial side of foot & Thumb walking & Shoulder/arm/hip/leg \\
Lateral side of foot & Thumb walking & Sciatic nerve \\
Back of ankle & Gentle pinching &
\end{tabular}

Finished with solar plexus on both feet.

lowed by light stretching of the foot or hand. From there, specific-finger pressure techniques are applied to the foot or hand following a protocol designed and supervised by a certified reflexologist (see Table 1 for sequence). All subjects received an equal number and duration of reflexology sessions.

\section{Control condition}

A 30-minute friendly visit was provided weekly by the certified reflexology practitioner. The visit began with 5 minutes of progressive relaxation ${ }^{28}$ techniques and was followed by 25 minutes of companionship and conversation.

There was no script for the reflexology or friendly visit sessions. During the reflexology sessions, it was normal for the practitioner to converse with the resident giving a brief overview of reflexology and answering any specific questions about the treatment. The resident would "normally" lead conversation and if a subject simply wanted to relax and close their eyes, then the reflexologist would encourage this. During the friendly visits, companionship and support was provided and included offering to water residents' plants or conversing about the weather or current events.

In order to provide for privacy and to reduce environmental stimuli, the intervention and control conditions were administered in each subject's private room. The door was closed (if possible) and the lights dimmed in order to provide privacy and avoid possible distractions. In preparation for, and during, the reflexology procedure, particular atten- tion was paid to the room condition, and the resident's preparation for and response to the intervention. These were noted in the reflexologist's journal. In addition, the reflexologist was trained to observe for episodes of stress and fatigue and agitation, to modify treatment to the subject's tolerance level, and to report the concerns to the nursing home staff.

\section{Data collection}

The data collection was performed using "gold standard" indices, and in a masked fashion by data collectors blind to group conditions. Each data collection encounter with a resident was designed to take approximately 30 minutes and was completed over four intervals across the day: (1) early morning: 7 AM-7:30 AM, (2) midmorning: $11 \mathrm{AM}-11: 30 \mathrm{AM}$, (3) early afternoon: 1 PM-1:30 PM, and (4) late afternoon: 3:30 PM-4 PM. These times were selected to maximize the opportunities to observe the resident affect and behavior and to capture diurnal variation in $\alpha$-amylase, while avoiding interruption of the interventionist's presence on the unit.

Over the course of the treatment day, an RN, specifically trained in observational and physiologic data collection techniques, collected four distinct types of data: (1) saliva samples from which salivary $\alpha$-amylase (sAA) was measured; (2) 5-minute observations of affect (e.g., anger, depression, anxiety) using the Apparent Affect Rating Scale (AARS), ${ }^{29,30}$ (3) pain using the checklist of nonverbal pain indicators (CNPI); ${ }^{31}$ and (4) other demographic and physiologic measures (e.g., blood pressure, pulse, mental status). 
Table 2. Baseline Characteristics of Sample $(\mathrm{n}=21)$

\begin{tabular}{lcc}
\hline & $\begin{array}{c}\text { Group } 1 \\
\mathrm{n}=10)\end{array}$ & $\begin{array}{c}\text { Group } 2 \\
(\mathrm{n}=11)\end{array}$ \\
\hline Age in years M(STD) & $87.2(7.8)$ & $88.6(8.6)$ \\
Length of stay in years M(STD) & $5.0(2.2)$ & $4.7(2.1)$ \\
Gender (female/male) & $8 / 2$ & $9 / 2$ \\
Case mix & $1.10(0.44)$ & $1.23(0.59)$ \\
Alpha amylase levels in U/mL M(STD) & $76.10(79.13)$ & $68.96(88.22)$ \\
Diastolic BP & $72.6(6.5)$ & $73.0(7.0)$ \\
Systolic BP & $121(9.9)$ & $121(10.3)$ \\
Pulse & $77.9(5.5)$ & $78.7(6.0)$ \\
MMSE score & $12.9(9.5)$ & $13.7(8.9)$ \\
Pain index & $4.2(2.73)$ & $3.7(3.29)$ \\
Observed emotion ratings M(STD) & & $5.0(2.7)$ \\
$\quad$ Anger & $4.7(1.8)$ & $6.1(2.7)$ \\
Anxiety & $6.5(3.7)$ & $14.8(4.1)$ \\
Alertness & $15.9(4.0)$ & $4.6(1.5)$ \\
High pleasure & $4.7(1.9)$ & $7.4(3.6)$ \\
Mild pleasure & $7.5(3.2)$ & $6.5(2.3)$ \\
Sadness & $5.9(2.0)$ & \\
\hline
\end{tabular}

M(STD), mean (standard deviation); BP, blood pressure; MMSE, Mini-Mental State Examination.

\section{Study outcomes}

Baseline data collection began in October 2005 and included completion of an intake assessment sheet of demographic information and the Folstein Mini-Mental State Examination (MMSE). ${ }^{32}$ Outcome measures were collected weekly over 8 weeks on the same day as the intervention.

Physiologic stress. The primary outcome of interest was distress, defined as the inability to adapt to a stressor, resulting in pathophysiologic processes. ${ }^{33}$ A stressor in this context was defined as the relationship between a challenge and an older person's appraisal of that challenge ${ }^{34}$ as reflected in responses that may be measured at the physiologic level. The most common of these responses involves the sympathoadrenal medullary system (SAM) ${ }^{35}$ When turned on, due to the anticipation or experience of an external challenge perceived as stressful, catecholamines are released from nerves and the adrenal medulla.

Assessment of SAM activity was only possible, until recently, via measurement of catecholamines in blood plasma or via electrophysiologic methods. ${ }^{36}$ Efforts to identify a less invasive and reliable method have led to the development of assays for salivary alpha amylase (sAA). sAA concentration is predictive of plasma catecholamine under a variety of stressful conditions, and is a more direct and simple endpoint of catecholamine activity than changes in heart rate. ${ }^{37}$ In addition, since sAA is unrelated to other biologic stress markers such as cortisol, it is considered a useful additional parameter for the measurement of stress. ${ }^{38-39}$

sAA possesses diurnal qualities, that is, levels shift through the course of the day. ${ }^{40-42}$ Thus, saliva samples (of $0.5-1.0 \mathrm{~mL}$ volume each) were collected in subjects within
30 minutes of awakening and every 3 hours thereafter (midmorning, early afternoon and late afternoon) to capture the circadian pattern. On each day, the collection procedures was identical. Care was taken when collecting saliva to avoid collection immediately after mouth cleaning, meals, snacks, or medications. The saliva samples were then transferred into 2 -mL cryovials and stored frozen (at least $-20^{\circ} \mathrm{C}$ ) until assayed. All samples were assayed for sAA using a highly sensitive enzyme immunoassay (Salimetrics, State College, PA).

Observation of affect. This was measured by AARS $^{29}$ four times over the course of the day: early morning, midmorning, early afternoon, and late afternoon, prior to the collection of each saliva sample. The scale consists of five items, requires 5 minutes of observation, and provides reliable and valid readings of depression, anxiety, anger, pleasure, and interest for both the cognitively intact and impaired. ${ }^{30}$ Psychometric properties have been well demonstrated in the sample population and documented in earlier studies, including inter-observer reliability (ICC $=0.92$ for the current study), convergent and discriminant validity, and support for its two-factor structure. ${ }^{30}$

Pain. The CNPI, a behavioral observation scale for nonverbal older adults with severe cognitive impairment, is one of the more rigorously tested pain assessment instruments. ${ }^{31}$ The CNPI is composed of six items that are rated as presence or absence of pain and has good face validity with verbal, horizontal visual, vertical visual, and faces pain scales, and established inter-rater reliability for periods of rest and movement. ${ }^{43,44}$ The CNPI was assessed prior to late morning saliva collection when residents were most likely to be awake, alert, and reactive. 
Table 3. Results of the Repeated-Measures Analysis of Variance

\begin{tabular}{llcrl}
\hline Outcome & \multicolumn{1}{c}{$\begin{array}{c}\text { Mean }(S D) \\
\text { control }\end{array}$} & $\begin{array}{c}\text { Mean }(S D) \\
\text { intervention }\end{array}$ & F & $\mathrm{p}$ \\
\hline Salivary $\alpha$-amylase & $(n=19) 86.45(72.35)$ & $23.88(61.94)$ & 4.37 & $\mathbf{0 . 0 4 9}$ \\
Diastolic blood pressure & $(n=21) 73.3(6.7)$ & $72.6(7.0)$ & 0.45 & 0.505 \\
Systolic blood pressure & $(n=21) 122(9.7)$ & $120(10.3)$ & 0.22 & 0.639 \\
Pulse & $(n=21) 78.7(5.9)$ & $77.1(5.1)$ & 0.02 & 0.893 \\
Observed emotion ratings & & & & \\
$\quad$ Anger & $(n=20) 5.1(2.3)$ & $6.5(1.9)$ & 1.40 & 0.251 \\
$\quad$ Anxiety & $(n=20) 6.7(3.7)$ & $15.6(4.3)$ & 1.83 & 0.192 \\
$\quad$ Alertness & $(n=20) 16.0(4.1)$ & $4.7(1.9)$ & 0.62 & 0.434 \\
$\quad$ Pleasure & $(n=20) 4.5(1.5)$ & $1.3(.84)$ & 4.06 & 0.444 \\
$\quad$ Sadness & $(n=21) 4.51(3)$ & $1.88(2.7)$ & 5.45 & 0.069 \\
Pain & & & $\mathbf{0 . 0 3 1}$ \\
\hline
\end{tabular}

Bold numbers are significant at $p<0.05$.

Additional measures. These included diastolic and systolic blood pressure (BP) and pulse intensity (HR) and cognitive assessment. The BP and HR assessments were collected following early afternoon saliva collection to minimize interference with AM care activities.

Cognitive assessment. This was conducted using Folstein's MMSE ${ }^{32}$ and was collected at baseline. The MMSE has demonstrated good test-retest reliability $(r=$ $0.80-0.95)$. It is traditionally used in tracking progressive declines in cognitive functioning in the nursing home population with dementia. The concurrent, convergent, and construct validity of the MMSE, with the Alzheimer's Disease Assessment Scale (ADAS), the Cambridge Examination for Mental Disorders of the Elderly (CAMCOG), the Clinical Global Impressions, and the Global Deterioration Scale has been well established. ${ }^{45}$

\section{Statistical analysis}

Data were analyzed using STATA version 9.0 (StataCorp LP, College Station, TX). Repeated-measures analysis of variance (ANOVA) with one between-subject effect (treatment condition) and one within-subject effect (time) was performed to determine whether there were statistically significant differences in outcomes measures. All analyses were by intention to treat (an analysis of only those who completed the study found no differences in results). Box's conservative epsilon correction was used to adjust for repeated measures.

\section{RESULTS}

Of the 48 residents approached for consent, 23 consented, 9 declined, 11 did not return consents, and 5 residents died while invitations to participate were in the mail. Of the 23 that consented, 2 were hospitalized prior to baseline data collection and were unable to participate, thus 21 completed the study. The types of dementia represented in the sample were:
Alzheimer's disease ( $\mathrm{n}=15,71 \%)$, dementia with Lewy bodies $(\mathrm{n}=3,14 \%)$, vascular dementia $(\mathrm{n}=2,9 \%)$, and frontotemporal dementia $(\mathrm{n}=1,5 \%)$. Time since diagnoses varied from 3 years to 7 years. The characteristics of the sample are demonstrated in Table 2. There was no statistically significant difference between the two groups at baseline.

Because of the agency's mission to serve poor Jewish elderly, the majority of the sample received Medicaid benefits $(80 \%)$ and was white $(100 \%)$, both much higher than national averages. Subjects ranged in age from 80 years to 96 years. Their average Medicaid case mix score (reflecting clinical complexity and cognitive, psychologic, and physical functioning) was 1.3, implying that residents were frailer than those found nationally in similarly sized facilities in metropolitan areas. In addition, most participants in the study had long lengths of stay; all of the subjects had been residents for 3 or more years. During the treatment and con-

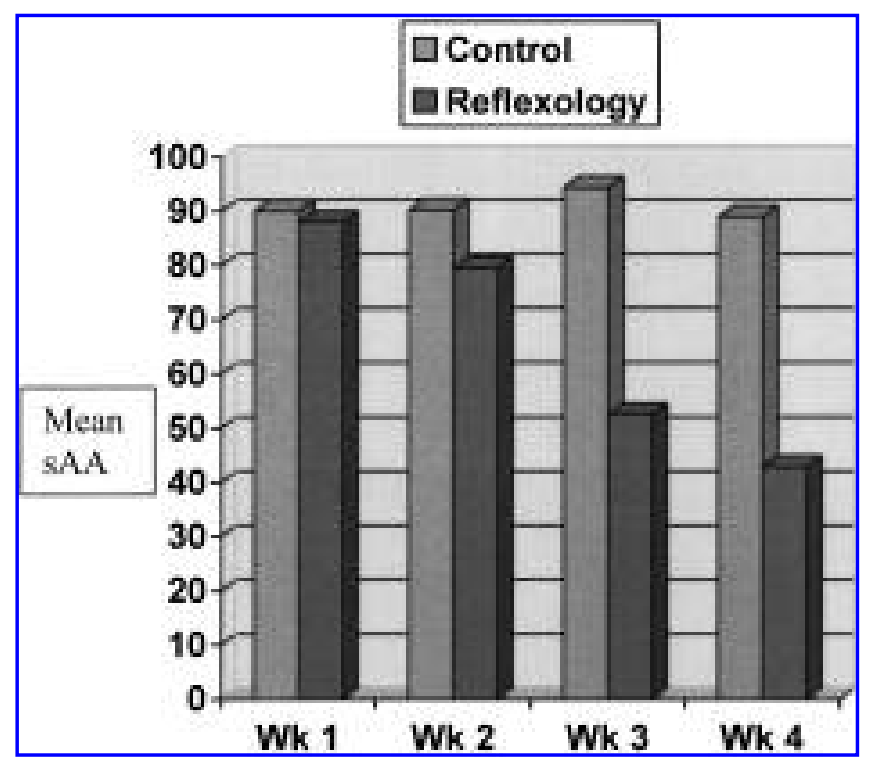

FIG. 1. Comparison of average salivary $\alpha$-amylase (sAA) levels over study period by treatment and control conditions $(n=21)$. 
trol conditions, no changes in medications or physiotherapies were recorded.

Table 3 presents the results of the repeated measures ANOVA. Statistically significant improvements for the outcome measures were demonstrated in the reflexology condition, while none were found to be significant in the control condition. Comparison of the outcome measures between the two conditions demonstrated a statistically significant decline in pain $(F=5.45, p=0.031)$, and sAA $(F=4.37, p=0.049)$, with borderline improvements in sadness $(F=4.06, p=0.069)$ when compared to the control condition.

Patterns in the differences between reflexology and control condition are suggested when the data are displayed graphically. As shown in Figure 1, for the control condition the average sAA values remained relatively stable. Yet sAA declined sharply over time for the reflexology condition, with the sharpest declines noted at weeks 3 and 4.

\section{DISCUSSION}

The results of this study indicated that residents receiving reflexology had clinically and statistically significant reductions in pain and sAA by the end of four weekly treatments. No improvements were noted in the control condition. The findings are remarkable given the small sample size and support preliminary evidence that reflexology treatment may be beneficial in the management of distress in nursing home residents with mild-to-moderate stage dementia. ${ }^{46}$

If reflexology is to be accepted as a discipline within conventional health care circles, then there needs to be a sound evidence base supporting the efficacy of the therapy. Critics of complementary therapy argue that placebo effects account for most of the therapeutic effect due to the compassion of the therapist or the relaxing atmosphere. In order to control for these conditions, both treatment and control conditions were provided to all subjects in the sample, in the same location, and by the same therapist. However, since only one reflexologist provided treatment, the positive results obtained from this single practitioner may not be reproducible across providers.

The study is limited in several important respects. The sample was homogeneous with respect to race and culture, with all residents being white, Jewish, and female. Because the sample was not randomly selected from the nursing home population, it is unclear whether the physiologic responses we observed here are generalizable to other groups. Although this study was much larger than most laboratorybased experiments of physiologic stress, analysis was hampered by sample size, precluding the ability to test interactions. As a result, we were unable to explore potentially important mediators and the stress response. In addition, sample size did not permit us to explore the interaction of affect and sAA in this sample.
These findings support preliminary evidence that reflexology treatment may be beneficial in the management of distress in nursing home residents with mild-to-moderate stage dementia. ${ }^{46}$

Recent evidence suggests that sAA may represent an objective neuroendocrine measure for changes in affect and pain intensity. ${ }^{39-42}$ Because reflexology seems to be of clinical usefulness for reducing distress, further clinical and laboratory studies into the mechanisms by which reflexology acts upon the sympathetic nervous system are warranted.

In conclusion, it is increasingly common for families of nursing home residents to ask for complementary therapies to supplement traditional care. ${ }^{47,48}$ Reflexology foot massage can be performed anywhere, requires no special equipment, is noninvasive, and does not interfere with patients' privacy. It is therefore critical for practitioners for have knowledge about complementary therapies; to alert residents and their families to possible contraindications with traditional medicines, and to be informed about research and practice guidelines related to complementary therapies.

\section{ACKNOWLEDGMENTS}

This study was funded by the Farber Family Foundation "Palliative Dementia Care" Grant.

\section{REFERENCES}

1. Chrousos GP, Gold PW. The concepts of stress and stress system disorders. JAMA 1992;267:1244-1252.

2. Remington R. Providing tender touch massage to elderly nursing home residents: A demonstration project. Geriatric Nurs 2002;21:303-308.

3. Rowe M, Alfred D. The effectiveness of slow stroke massage in diffusing agitated behaviors in individuals with Alzheimer's disease. J Gerontol Nurs 1999;25:22-34.

4. Snyder M, Egan EC, Kenneth R, Burns KR. Efficacy of hand massage in decreasing agitation behaviors associated with care activities in persons with dementia. Geriatr Nurs 1995;16: 60-63.

5. Woods DL, Dimond M. The effect of therapeutic touch on agitated behavior and cortisol in persons with Alzheimer's disease. Biol Res Nurs 2002;4:104-114.

6. Kim EJ, Buschmann MT. The effect of expressive physical touch on patients with dementia. Int J Nurs Stud 1999;36: 235-243.

7. McCann K, McKenna HP. An examination of touch between nurses and elderly patients in a continuing care setting in Northern Ireland. J Adv Nurs 1993;18:838-846.

8. Lafferty WE, Downey L., McCarty, RL, et al. Evaluating CAM treatment at the end of life: A review of clinical trials for massage and meditation. Compl Ther Med 2006;14:100-112.

9. Sherman KJ, Dixon MW, Thompson D, Cherkin D. Development of a taxonomy to describe massage treatments for musculoskeletal pain. BMC Compl Alt Med 2006;6:24. 
10. Mackereth P, Dryden SF, Franekl B. Reflexology: Recent research approaches. Complement Ther Nurs Midwifery 2000;6:66-71.

11. Richardson J. The use of randomized control trials in complementary therapies: Exploring the issues. J Adv Nurs 2000; 32:P398-P406.

12. Richard J, Picot A, de Bus P, et al. Indications for relaxation in geriatrics. Ann Med Psych 1975;2:703-721.

13. Norber A, Melin E, Asplund K. Reactions to music, touch and object presentation in the final stage of dementia: An exploratory study. Int J Nurs Stud 1986;23:315-323.

14. Grant P, Shinkle R, Lipps G. The impact of an inter-institutional touch therapies on nursing home residents requiring a high level of care. Gerontologist 1992;32:834-842.

15. Gleeson M, Timments F. The use of touch to enhance nursing care of older person in long term mental health care facilities. J Psych Ment Health Nurs 2004;11:541-545.

16. Brooker DJ, Snape M, Johnson E, et al. Single case evaluation of the effects of aromatherapy and massage on disturbed behavior in severe dementia. Br J Clin Psych 1997;36:287296.

17. Kilstoff K, Chenoweth L. New approaches to health and wellbeing for dementia day-care clients, family carers and day-care staff. Int J Nurs Prac 1998;4:70-83.

18. Sansone P, Schmitt L. Providing tender touch massage to elderly nursing home residents: A demonstration project. Geriatr Nurs 2000;21:303-308.

19. Crane B. Reflexology: The Definitive Practitioner's Manual. London Element Books Limited, 1997.

20. Kuhn MA. Complementary Therapies for Health Care Providers. Philadelphia: Lippincott, Williams, and Wilkins, 1999.

21. Oleson T, Flocco W. Randomized controlled study of premenstrual symptoms treated with ear, hand and foot reflexology. Obstet Gynecol 1993;82:906-911.

22. Launso L, Brendstrup E, Arnberg S. An exploratory study of reflexological treatment for headache. Alt Ther Health Med 1999;5:57-65.

23. Siev-Ner I, Gamus D, Lerner-Geva L, Achiron A. Reflexology treatment relieves symptoms of multiple sclerosis: A randomized controlled study. Mult Scler 2003;9:356-361.

24. Solà I, Thompson E, Subirana M, et al. Non-invasive interventions for improving well-being and quality of life in patients with lung cancer. Cochrane Database Syst Rev 2004;18:CD004282.

25. Fellowes D, Barnes K, Wilkenson S. Aromatherapy and massage for symptom relief in patients with cancer. Cochrane Database Syst Rev 2004;2:CD002287.

26. Bardia A, Barton DL, Prokop LJ, et al. Efficacy of complementary and alternative medicine therapies in relieving cancer pain: A systematic review. J Clin Oncol 2006;24:5457-5464.

27. Reisberg B. Functional assessment staging (FAST). Psychopharmacol Bull 1988;24:653-659.

28. Welden S, Yesavage JA. Behavioral improvement with relaxation training in senile dementia. Clin Gerontol 1982;1:45-49.

29. Lawton MP, Van Haitsma K, Perkinson K, et al. Observed affect and quality of life in dementia: Further affirmations and problems. J Ment Health Aging 1999;5:69-81.

30. Lawton MP, Van Haitsma KS, Klapper JA. Observed affect in nursing home residents. J Gerontol B Psychol Sci 1996;51B:3-14.
31. Nygaard HA, Jarland M. The Checklist of Nonverbal Pain Indicators (CNPI): Testing of reliability and validity in Norwegian nursing homes. Age Ageing 2006;35:79-81.

32. Folstein M, Folstein S, McHugh P. Mini-Mental State: A practical method for grading the cognitive state of patients for the clinician. J Psychiatr Res 1975;12:189-198.

33. Selye H. Stress Without Distress. New York: Signet, 1975.

34. Lazarus RS, Folkman S. Stress, Appraisal, and Coping. New York: Springer, 1984.

35. McEwan BS. Protective and damaging effects of stress mediators. NEJM 1998;358:171-179.

36. Granger DA, Kivlighan KT, El-Sheikh M, et al. Salivary $\alpha$ amylase in biobehavioral research: Recent developments and applications. Ann NY Acad Sci 2007;1098:122-144.

37. Rohleder N, Nater UM, Wolf JM, et al. Psychosocial stressinduced activation of salivary alpha-amylase: An indicator of sympathetic activity? Clin Phys 1996;16:433-448.

38. Nater UM, Rohleder N, Scholotz W, et al. Stress-induced changes in human salivary alpha-amylase activity associations with adrenergic activity. Psychoneuroendocrinology 2006;31: 49-58.

39. Nater UM, Rohleder N, Gaab J, et al. Human salivary alphaamylase reactivity in a psychosocial stress paradigm Int J Psychophysiol 2005;55:333-342.

40. Nater UM, Rohleder N, Schlotz W, et al. Determinants of the diurnal course of salivary alpha-amylase. Psychoneuroendocrinology 2007;32:392-401.

41. Shirasaki S, Fujii H, Takahashi M, et al. Correlation between salivary alpha-amylase activity and pain scale in patients with chronic pain. Regional Anesthes Pain Med 2007;32:120-123.

42. van Stegeren A, Rohleder N, Everaerd W, Wolf OT. Salivary alpha amylase as marker for adrenergic activity during stress: Effect of beta blockade. Psychoneuroendocrinology 2006;31: 137-141.

43. Herr K, Bjoro K, Decker S. Tools for assessment of pain in nonverbal older adults with dementia: A state of the science review. J Pain Symptom Manage 2006;31:170-192.

44. Sandra MG, Zwakhalen PH, Hamers L, et al. Pain in elderly people with severe dementia: A systematic review of behavioural pain assessment tools BMC Geriatr 2006;6:3.

45. Korner A, Lauritzen L, Bech P. A psychometric evaluation of dementia rating scales. Eur Psychiatry 1996;11:185-191.

46. Meng G, Zhao L. Foot reflexology therapy in senile dementia: Report of 2 cases. 1993 China Reflexology Symposium Report, Beijing, China Reflexology Association, p. 14.

47. McBee L, Lantz M. Complementary and alternative medicine interventions for nursing home residents. Clin Geriatr 2003;11: 34-36.

48. Routasalo P. Non necessary touch in the nursing care of elderly people. J Adv Nurs 1996;23:904-911.

Address reprint requests to: Nancy A. Hodgson, Ph.D., R.N. Center for Applied Research on Aging and Health Thomas Jefferson University 130 South 9th Street, Suite 512 Philadelphia, PA 19107

E-mail: nancy.hodgson@jefferson.edu 